\title{
Features of infective native aortic aneurysms on computed tomography
}

\author{
Warissara Jutidamrongphan ${ }^{1}$, Boonprasit Kritpracha² ${ }^{2}$ Karl Sörelius ${ }^{3}$, Keerati Hongsakul ${ }^{1}$ and \\ Ruedeekorn Suwannanon ${ }^{1 *}$ (D)
}

\begin{abstract}
Background: Infective native aortic aneurysm (INAA) is a rare clinical diagnosis. The purpose of this study was to describe the CT findings of INAAs in detail.

Methods: This was a retrospective single-center study of INAA patients at a major referral hospital between 2005 and 2020. All images were reviewed according to a protocol consisting of aneurysm features, periaortic findings, and associated surrounding structures.

Results: One hundred and fourteen patients (mean age, 66 years [standard deviation, 11 years]; 91 men) with 132 aneurysms were included. The most common locations were infrarenal (50.8\%), aortoiliac (15.2\%), and juxtarenal (12.9\%). The mean transaxial diameter was $6.2 \mathrm{~cm}$. Most INAAs were saccular (87.9\%) and multilobulated (91.7\%). Calcified aortic plaque was present in $93.2 \%$ and within the aneurysm in 51.5\%. INAA instability was classified as contained rupture (27.3\%), impending rupture (26.5\%), and free rupture (3.8\%). Rapid expansion was demonstrated in 13 of 14 (92.9\%) aneurysms with sequential CT studies. Periaortic inflammation was demonstrated as periaortic enhancement (94.7\%), fat stranding (93.9\%), soft-tissue mass (92.4\%), and lymphadenopathy (62.1\%). Surrounding involvement included psoas muscle (17.8\%), spondylitis (11.4\%), and perinephric region (2.8\%). Twelve patients demonstrated thoracic and abdominal INAA complications: fistulas to the esophagus (20\%), bronchus (16\%), bowel (1.9\%), and inferior vena cava (IVC) (0.9\%).
\end{abstract}

Conclusion: The most common CT features of INAA were saccular aneurysm, multilobulation, and calcified plaques. The most frequent periaortic findings were enhancement, fat stranding, and soft-tissue mass. Surrounding involvement, including psoas muscle, IVC, gastrointestinal tract, and bronchi, was infrequent but may develop as critical INAA complications.

Keywords: Infective native aortic aneurysm, Aortic aneurysm, Computed tomography, Mycotic aortic aneurysm, Infected aortic aneurysm

\section{Key points}

- The characteristic INAA features were multilobulated saccular aneurysm with periaortic abnormality.
- Presence of calcified atherosclerotic plaque made INAA indistinguishable from degenerative aneurysm.

- Psoas muscle involvement and fistulas were rare but may represent critical complications.

\footnotetext{
*Correspondence: sruedeek@medicine.psu.ac.th; Sruedeekorn@gmail.com

${ }^{1}$ Department of Radiology, Faculty of Medicine, Prince of Songkla

University, 15 Karnjanavanich Rd., Hat Yai, Songkhla 90110, Thailand

Full list of author information is available at the end of the article
} 


\section{Introduction}

Infective native aortic aneurysm (INAA), also known as mycotic aortic aneurysm, is a challenging disease in respect of making the diagnosis $[1,2]$. It is a rare entity found in only $0.6-2.6 \%$ of all aortic aneurysms in western countries and up to $13 \%$ in Asia [3, 4]. INAA may develop through various pathophysiological ways according to the amended Wilson's classification [5, 6]: (1) due to septic emboli lodging in the aortic wall from infective endocarditis; (2) blood-borne bacteria inoculated in the aortic wall during bacteremia; (3) infection of a preexisting aneurysm due to blood-borne bacteria; and (4) aneurysms developing in patients with advanced human immunodeficiency virus infection.

Due to INAA variability and non-specific manifestations, a delayed or missed diagnosis may lead to a fatal outcome. Therefore, making an accurate and timely diagnosis is crucial for proper management, including securement of the microbiological specimen, initiation of appropriate antibiotic therapy, and surgery.

The European Society for Vascular Surgery 2019 guidelines [7] on abdominal aortic aneurysms recommend that the diagnostic workup of INAA should consist of a combination of: (1) clinical presentation; (2) laboratory results; and (3) computed tomography (CT) findings. Since there is no pathognomonic symptom or test for the disease, CT is thus the cornerstone for the diagnosis of
INAA. Typical CT findings have been reported to consist of saccular shape, multilobulated or eccentric aneurysm, periaortic gas, soft-tissue mass, rapid expansion (days) or rupture or both, atypical location (e.g., para-visceral), and multiple aneurysms in different areas $[7,8]$.

However, several causes of aortitis might also mimic INAA identification on CT images [9]. Moreover, detailed studies on CT findings of INAA are scarce and small partly due to the rarity of the disease and partly due to the fact that CT findings have been reported mainly as a subordinate finding in studies reporting treatment outcome [2].

This study aimed to describe the features of INAA on $\mathrm{CT}$ at presentation, including details of the aneurysm, presence of aortic wall calcification, and periaortic findings, as well as associated findings in other organs.

\section{Materials and methods}

This is a retrospective study of patients treated for INAA at a major referral center between September 2005 and April 2020. Patients were identified from the internal hospital registry in the vascular surgery and radiology databases. The institutional review board of the Faculty of Medicine, Prince of Songkla University approved the study. Informed consent was not required due to the retrospective nature of the study. The $\mathrm{CT}$ examinations were performed on a 64-slice multidetector computed

\section{Patients diagnosed with aortic aneurysms or aortitis between Sept. 2005 and May 2020 $(n=3660)$}

Excluded due to incomplete fulfillment of criteria for INAA $(n=3542)$

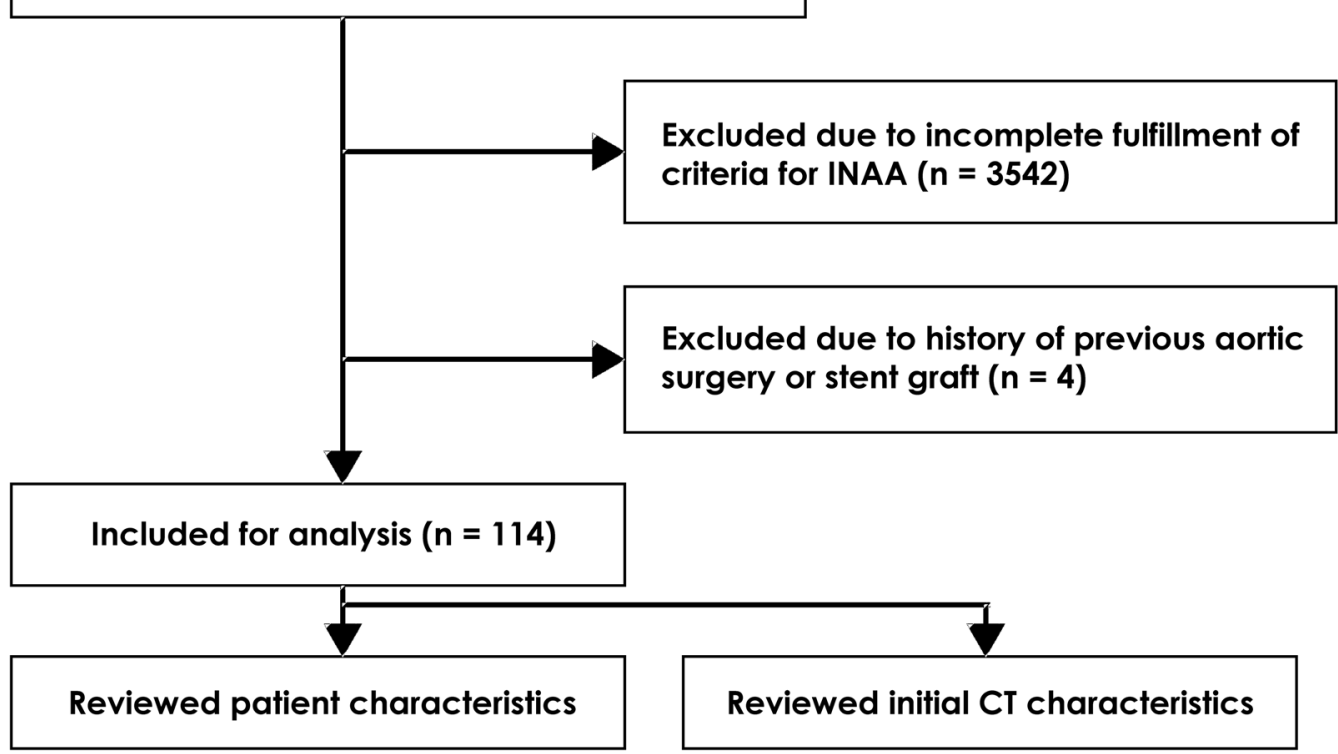

Fig. 1 Flow diagram of this study 
Table 1 Characteristics in INAA patients $(n=114)$

\begin{tabular}{|c|c|}
\hline Variable & $n(\%)$ \\
\hline Age $\left(\right.$ year) ${ }^{\mathrm{a}}$ & $66(11)$ \\
\hline Male & $91(79.8)$ \\
\hline \multicolumn{2}{|l|}{ Medical history } \\
\hline Hypertension & $58(50.9)$ \\
\hline Diabetes mellitus & $40(35.1)$ \\
\hline Ischemic heart disease & $10(8.8)$ \\
\hline Renal failure & $10(8.8)$ \\
\hline HIV infection & $6(5.3)$ \\
\hline Cerebrovascular disease & $5(4.4)$ \\
\hline COPD & $3(2.6)$ \\
\hline Steroid treatment & $3(2.6)$ \\
\hline \multicolumn{2}{|l|}{ Clinical manifestation } \\
\hline Abdominal pain & $86(75.4)$ \\
\hline Back pain & $21(18.4)$ \\
\hline Fever & $95(83.3)$ \\
\hline Circulatory shock & $10(8.8)$ \\
\hline Hemoptysis & $5(4.4)$ \\
\hline UGIB & $6(5.3)$ \\
\hline LGIB & $3(2.6)$ \\
\hline Urinary tract infection & $13(11.4)$ \\
\hline Septic arthritis & $7(6.1)$ \\
\hline Abscess & $5(4.4)$ \\
\hline Pulmonary tuberculosis & $4(3.5)$ \\
\hline Enterocolitis & $2(1.8)$ \\
\hline Osteomyelitis & $1(0.9)$ \\
\hline \multicolumn{2}{|l|}{ Laboratory data } \\
\hline Leukocytosis & $107(93.9)$ \\
\hline Elevated ESR & $59(51.8)$ \\
\hline Elevated CRP & $59(51.8)$ \\
\hline Positive blood culture & $40(35.1)$ \\
\hline Salmonella & $15(13.2)$ \\
\hline Staphylococcus & $8(7)$ \\
\hline Streptococcus & $6(5.3)$ \\
\hline Melioidosis & $5(4.4)$ \\
\hline Enterococcus coli & $5(4.4)$ \\
\hline Mycobacterium & $2(1.8)$ \\
\hline Other & $1(0.9)$ \\
\hline
\end{tabular}

INAA infective native aortic aneurysm, HIV human immunodeficiency virus, $C O P D$ congestive obstructive pulmonary disease, $U G I B$ upper gastrointestinal bleeding, $L G I B$ lower gastrointestinal bleeding, ESR erythrocyte sedimentation rate, $C R P$ C-reactive protein

${ }^{\text {a }}$ Data are mean with standard deviation in parenthesis

tomography (MDCT) scanner from 2015 to 2018, a 160slice MDCT scanner from 2015, and a 512-slice MDCT scanner from 2018.

The diagnostic workup was performed according to the newly proposed algorithm in the European Journal of Vascular and Endovascular Surgery [7]. The diagnostic algorithm was a combination of three clinical criteria: (1) clinical presentation; (2) laboratory results; and (3) CT imaging. All patients in this study had to meet all three of the criteria for a definite diagnosis to be included in this study. Patients with a history of previous aortic surgery or an incomplete/inadequate initial CT study were excluded.

The retrospective review of all patients included demographic data on patient characteristics (i.e., sex, age, medical history including immunodeficiency and comorbidities, treatments causing relative immunodeficiency, symptoms [pain, fever, shock], concurrent infection), laboratory and microbiological findings (inflammatory markers: C-reactive protein, levels of leukocytes, cultures from blood/tissues, results of a polymerase chain reaction assay for bacterial identification), and detailed CT findings.

\section{CT analysis}

All CT examinations were reviewed by a senior consultant in cardiovascular radiology with 8 years of experience and an in-training resident with 3 years of experience at the same time to make the consensus. Despite being aware that all images were of INAA, the reviewers were blinded to the clinical information of each patient.

The CT examinations were evaluated on three categories. First were the features of the aneurysm that included the number of aneurysms (single or multiple), location, size (measured in maximum transaxial diameter $(\mathrm{cm})$ of outer wall to outer wall of aneurysm including periaortic soft tissue), rupture status (divided into no rupture, impending rupture, contained rupture, free rupture), rapid progression, shape (saccular or fusiform), contour (multilobulated or non-lobulated), and the presence of calcified atherosclerotic plaque. The extent of calcification was classified into five types based on the findings in this study: type 0 saccular aneurysm without calcification; type 1 saccular aneurysm with calcified aneurysm neck; type 2 saccular aneurysm with calcification of the aorta more than $1 \mathrm{~cm}$ from the aneurysm; type $3 \mathrm{saccu}$ lar aneurysm with calcification within the aneurysm wall; type 4 fusiform aneurysm with calcified intima; and type 5 fusiform aneurysm with peripheral calcification. Second were the periaortic findings that included aneurysmal wall enhancement, fat stranding, soft-tissue mass, lymphadenopathy, fluid, and the presence of ectopic gas. Third was the associated surrounding organ involvement according to either the overall or specific location of the INAA (e.g., abdominal aorta and thoracic aorta), such as psoas muscle, perinephric region, aortocaval fistula, the hollow viscus (bowel, bronchus, esophagus), and spondylitis. 

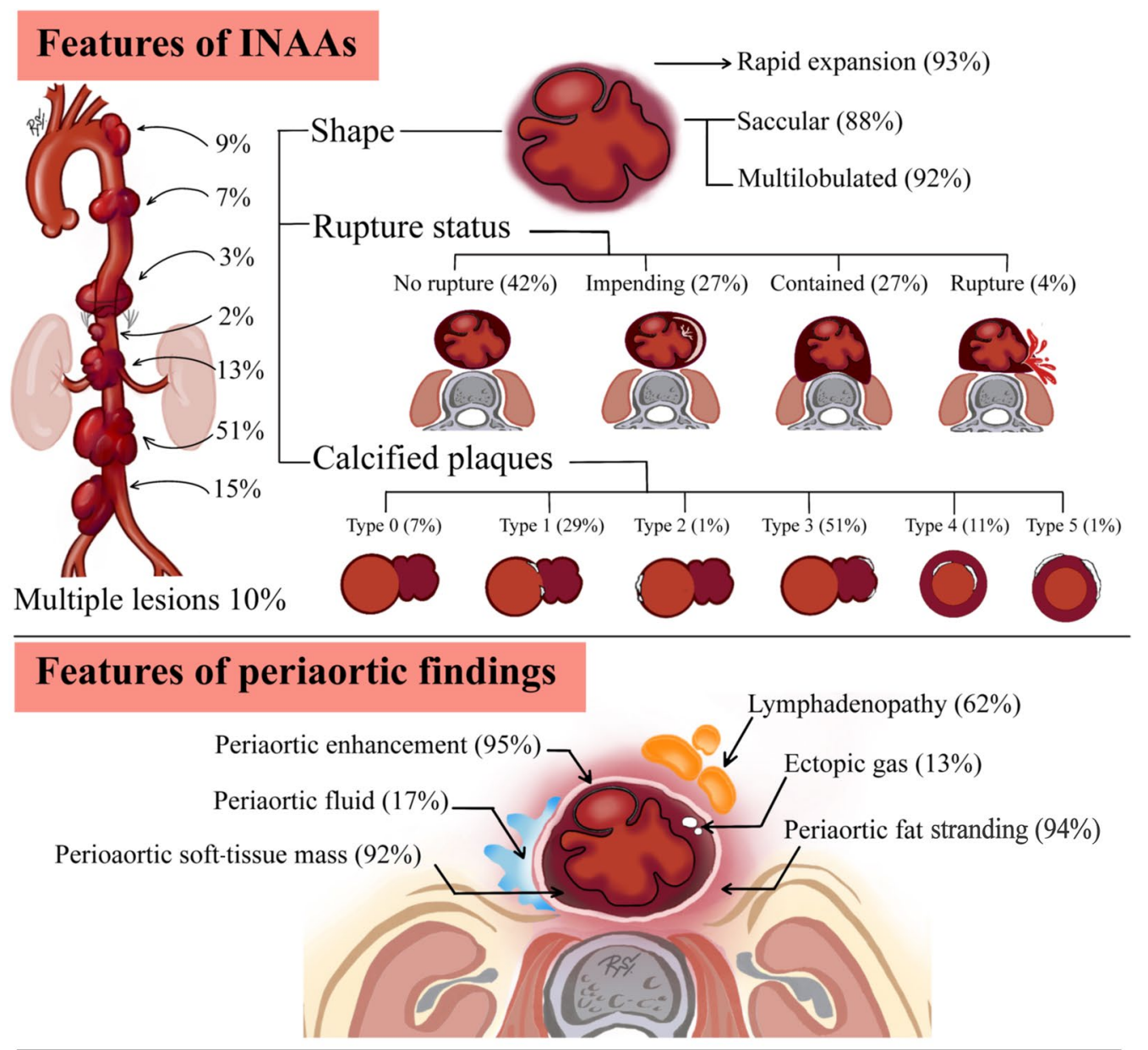

\section{Features of surrounding organ involvement ${ }^{*}$}

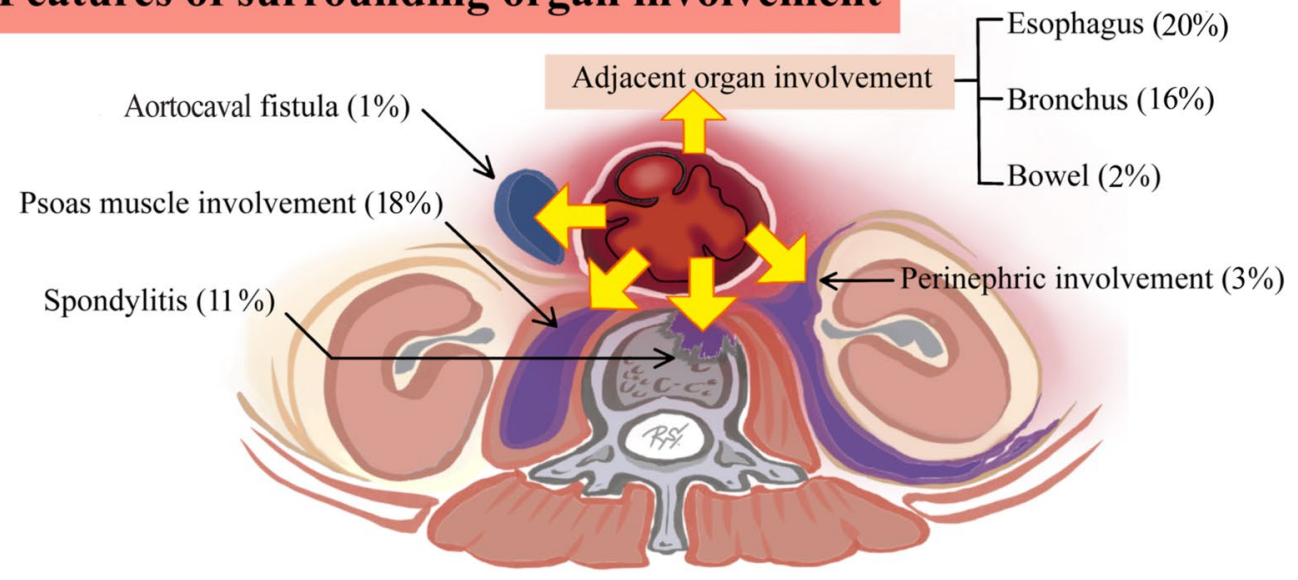

Fig. 2 Summary of all CT findings in INAA in 132 aneurysms in three catagories: (1) features of infected aneurysm; (2) periaortic findings; and (3) surrounding organ involvement. Asterisk $\left(^{*}\right)$ indicates surrounding organ involvement according to the anatomical structure and location of INAA

\section{Statistical analysis}

The sample size was calculated by the infinite population proportion formula, which revealed at least 100 subjects to represent justification of the sample size. All recorded data were assessed for normality with histograms. Continuous data are expressed as mean and 
Table 2 Background and features of 132 aneurysms on initial CT in 114 patients with INAA

\begin{tabular}{ll}
\hline Patients & $\mathbf{n}=\mathbf{1 1 4}$ \\
\hline Single aneurysm & $102(89.5)$ \\
Multiple aneurysms & $12(10.5)$ \\
CT findings of aneurysms $(n=132)$ & $n(\%)$ \\
Location & \\
Arch & $12(9.1)$ \\
Descending aorta & $9(6.8)$ \\
Thoracoabdominal & $4(3)$ \\
Suprarenal & $3(2.3)$ \\
Juxtarenal & $17(12.9)$ \\
Infrarenal & $67(50.8)$ \\
Aortoiliac & $20(15.2)$ \\
Size (cm) & \\
Rupture status & $6.2(2.3)$ \\
No rupture & \\
Impending rupture & $56(42.4)$ \\
Contained rupture & $35(26.5)$ \\
Rupture & $36(27.3)$ \\
Shape & $5(3.8)$ \\
Saccular & \\
Fusiform & $116(87.9)$ \\
Multilobulated contour & $16(12.1)$ \\
Rapid expansion & \\
Calcified atherosclerotic plaque & $121(91.7)$ \\
Type 0 & $13(92.9)$ \\
Type 1 & \\
Type 2 & $9(6.8)$ \\
Type 3 & $38(28.8)$ \\
Type 4 & $1(0.8)$ \\
Type 5 & $68(51.5)$ \\
\hline NAA Infctivenative & $14(10.6)$ \\
\hline & $2(1.5)$ \\
\hline
\end{tabular}

INAA Infective native aortic aneurysm

a Data are mean with standard deviation in parenthesis

${ }^{\mathrm{b}}$ Data represent 13 of 14 patients with sequential CT studies

standard deviation (SD) and categorical variables as proportions (\%). Non-normally distributed continuous data are expressed as median and range. All statistical analyses were performed by a statistician using $\mathrm{R}$ software, version 4.0.3 (https://www.r-project.org/).

\section{Results}

\section{Patient characteristics}

During the study period, 3660 patients were either diagnosed with aortic aneurysms $(n=3537)$ or aortitis $(n=123)$. Among these patients, 3546 patients were excluded, leaving 114 patients who fulfilled all criteria for INAA. The study flow diagram is shown in Fig. 1. The majority of the patients were male $(91 / 114 ; 79.8 \%)$ and the mean (SD) age was 66 (11) years (range 41-92 years).
The patient characteristics are provided in Table 1. Twenty-nine $(25.4 \%)$ patients had at least one concomitant infection, documented as urinary tract infection $(n=13)$, septic arthritis $(n=7)$, abscess $(n=5)$, pulmonary tuberculosis $(n=4)$, enterocolitis $(n=2)$, and osteomyelitis $(n=1)$. Forty $(35.1 \%)$ cases had positive blood cultures. Almost half of the patients $(47 / 114 ; 41.2 \%)$ had antibiotic administration prior to obtaining the blood cultures at this major hospital, and only a small number $(7 / 47 ; 14.9 \%)$ of them had positive culture results. Since most of the patients $(99 / 114 ; 86.8 \%)$ were referred from surrounding primary hospitals, more than one-third (39/99; 39.4\%) had documented previous antibiotic therapy, while such data were unavailable in approximately half of them (45/99; 45.5\%).

\section{Features of infective native aortic aneurysm on CT}

The CT findings were divided into three categories: (1) features of the infected aneurysm; (2) periaortic tissues; and (3) associated structures (Fig. 2).

\section{Features of the infective aneurysm}

The distributions of INAA were in the aortic arch (12/132; 9.1\%), descending aorta (9/132; 6.8\%), thoracoabdominal aorta $(4 / 132 ; 3 \%)$, suprarenal aorta (3/132; $2.3 \%)$, juxtarenal aorta $(17 / 132 ; 12.9 \%)$, infrarenal aorta in half of the patients $(67 / 132 ; 50.8 \%)$, and aortoiliac location (20/132; 15.2\%). In 12 (10.5\%) patients, multiple aneurysms were found (Table 2).

Of the 132 aneurysms, the types of aneurysms found were saccular $(116 / 132 ; 87.9 \%)$ and fusiform (16/132; $12.1 \%)$, while most of them showed a multilobulated contour (121/132; 91.7\%). The mean (SD) aneurysmal sac size measured in the maximal transaxial diameter was $6.2 \mathrm{~cm}(2.3 \mathrm{~cm})$. In $56(42.4 \%)$ of the 132 aneurysms, no sign of rupture (Fig. 3a) was identified. The CT findings of aneurysm instability in this study were impending rupture (Fig. 3b) in 35 (26.5\%) aneurysms, contained rupture (Fig. 3c) in 36 (27.3\%) aneurysms, and free rupture (Fig. 3d) in 5 (3.8\%) aneurysms. In the patients with sequential CT studies performed, the median values of rapid expansion in size and time of the aneurysms were $1.1 \mathrm{~cm}$ (range $0.3-4 \mathrm{~cm}$ ) and 69 days (range $5-136$ days), respectively, found in 13 of 14 patients (92.9\%). Almost all INAA (123/132; 93.2\%) in this study demonstrated associated calcified aortic plaques that were classified into five types (Table 2). The most common type found in half of the patients was type 3 saccular aneurysm with calcification within the aneurysm wall $(68 / 132 ; 51.5 \%)$ while the second and third most common types were type 1 saccular aneurysm with calcified aneurysm neck 


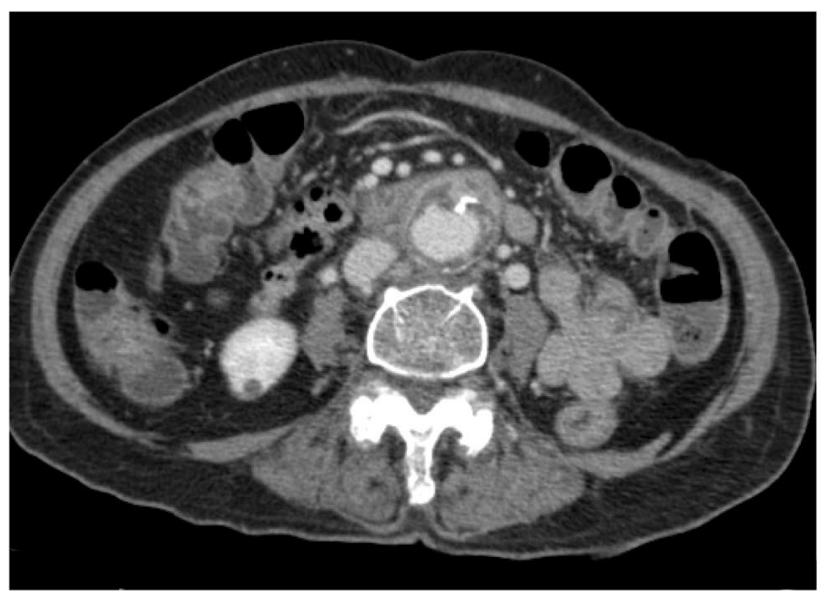

a.

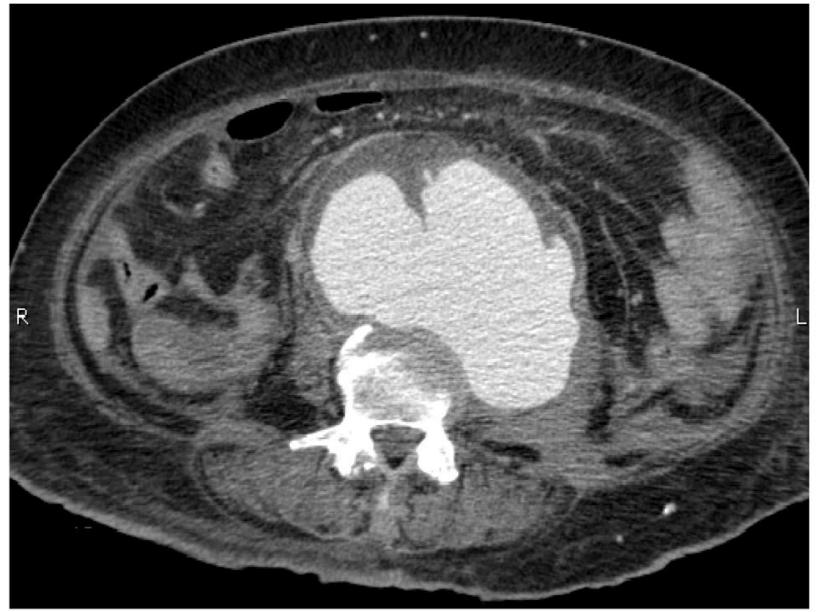

c.

Fig. 3 CT findings of INAA instability. a Axial CECT image of a 77-year-old female patient reveals an infrarenal aneurysm and periaortic soft-tissue mass without signs of instability. $\mathbf{b}$ Axial NECT image of a 68-year-old male patient depicts hyperattenuation of the peripheral thrombus in an infrarenal aneurysm indicating impending rupture. c Axial CECT of a 61-year-old female patient demonstrates a multilobulated infrarenal aneurysm with its posterior wall draping along the anterior vertebral body, draped aorta sign, representing contained rupture. $\mathbf{d}$ Coronal MIP angiographic CT image of a 63-year-old male patient reveals active contrast extravasation from the infrarenal INAA into the retroperitoneal cavity indicating free rupture. INAA infective native aortic aneurysm, CECT contrast-enhanced computer tomography, NECT non-enhanced computed tomography, MIP maximum intensity projection

\section{(See figure on next page.)}

Fig. 4 Anatomical classification of the INAA with associated calcified aortic plaques: type 0 saccular aneurysm without calcification; type 1 saccular aneurysm with calcified aneurysm neck; type 2 saccular aneurysm with aortic calcification far from aneurysm (more than $1 \mathrm{~cm}$ ); type 3 saccular aneurysm with calcification within aneurysm wall; type 4 fusiform aneurysm with calcified intima; and type 5 fusiform aneurysm with peripheral calcification. Yellow arrows indicate calcified plaque on CT

(38/132; $28.8 \%)$ and type 4 fusiform aneurysm with calcified intima (14/132; 10.6\%), respectively (Fig. 4).
Features of the periaortic tissues on CT

Periaortic enhancement (125/132; 94.7\%), fat stranding (124/132; 93.9\%), and soft-tissue mass (122/132; 92.4\%) were the most common periaortic findings (Table 3 and 


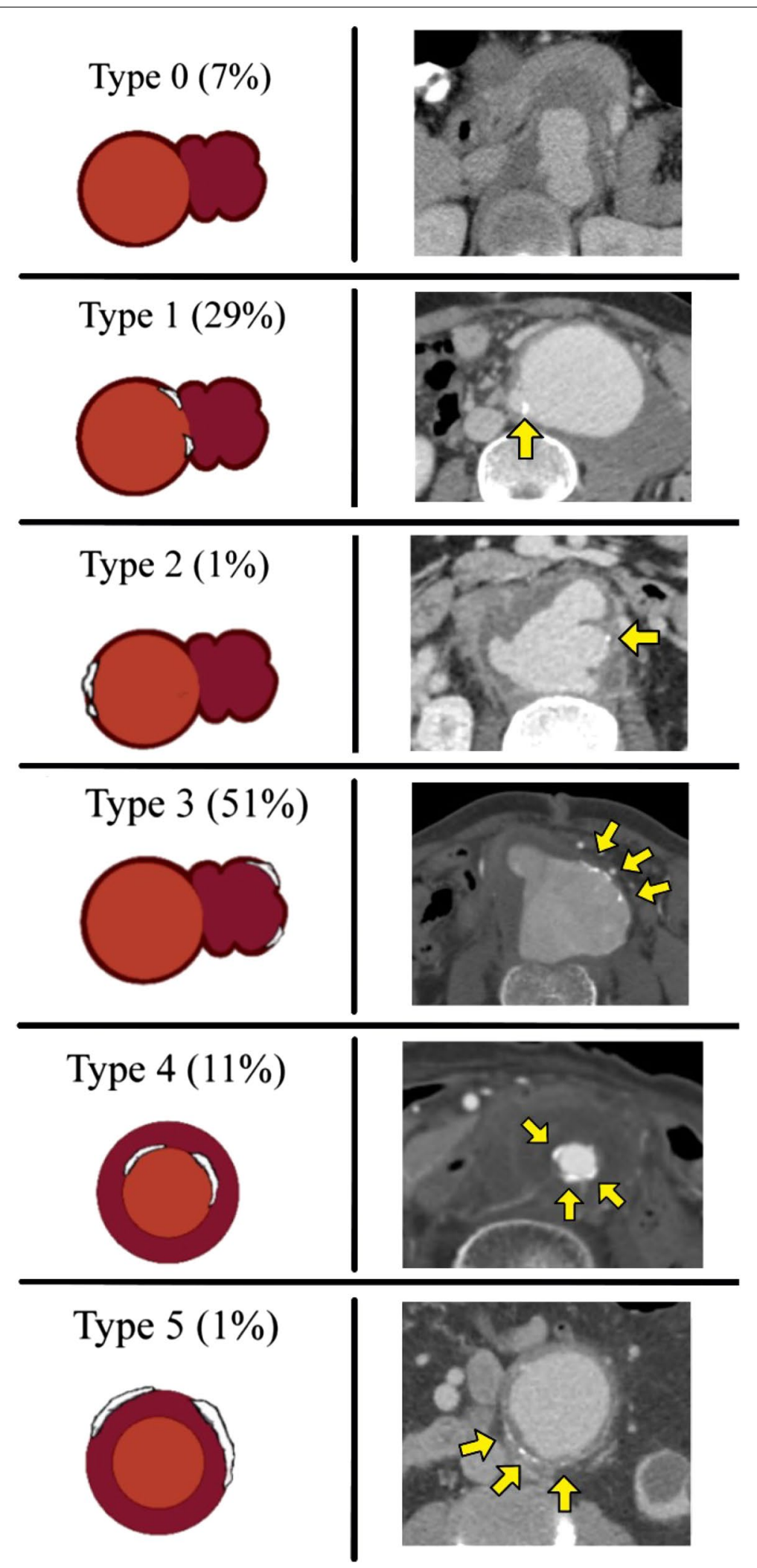

Fig. 4 (See legend on previous page.) 
Table 3 Periaortic findings of aneurysms on initial CT in INAA patients

\begin{tabular}{lr}
\hline CT findings of aneurysms $(\boldsymbol{n}=\mathbf{1 3 2})$ & $\boldsymbol{n}(\mathbf{\%})$ \\
\hline Periaortic enhancement & $125(94.7)$ \\
Periaortic fat stranding & $124(93.9)$ \\
Periaortic soft-tissue mass & $122(92.4)$ \\
Periaortic lymphadenopathy & $82(62.1)$ \\
Periaortic fluid & $22(16.7)$ \\
Presence of ectopic gas & $17(12.9)$ \\
\hline
\end{tabular}

Data are presented as number (\%)

INAA infective native aortic aneurysm

Fig. 5a). About two-thirds of the patients (82/132; 62.1\%) demonstrated periaortic lymphadenopathy (Fig. 5b).
The less frequent features were periaortic fluid (22/132; $16.7 \%$ ) and ectopic gas (17/132; 12.9\%) (Fig. 5c, d).

\section{Features demonstrated in the surrounding organs on CT}

Overall, the INAAs were classified according to anatomical location as thoracic aortic aneurysms $(25 / 132 ; 18.9 \%)$ and abdominal aortic aneurysms (107/132; 81.1\%). In the intra-abdominal region, the two most common surrounding structure involvements were psoas muscle $(19 / 107 ; 17.8 \%)$ and perinephric region $(3 / 107 ; 2.8 \%)$ (Table 4, Fig. 6a, b). Aortocaval fistula was rarely found $(1 / 107 ; 0.9 \%)$ (Fig. 6c). Hollow viscus involvement, such as the bowel $(2 / 107 ; 1.9 \%)$ (Fig. $6 \mathrm{~b})$, was scarce in the abdomen but appeared relatively more prevalent in the thorax as suspected esophageal (Fig. 6d) and bronchial complications, up to $20 \%(5 / 25)$ and $16 \%(4 / 25)$,

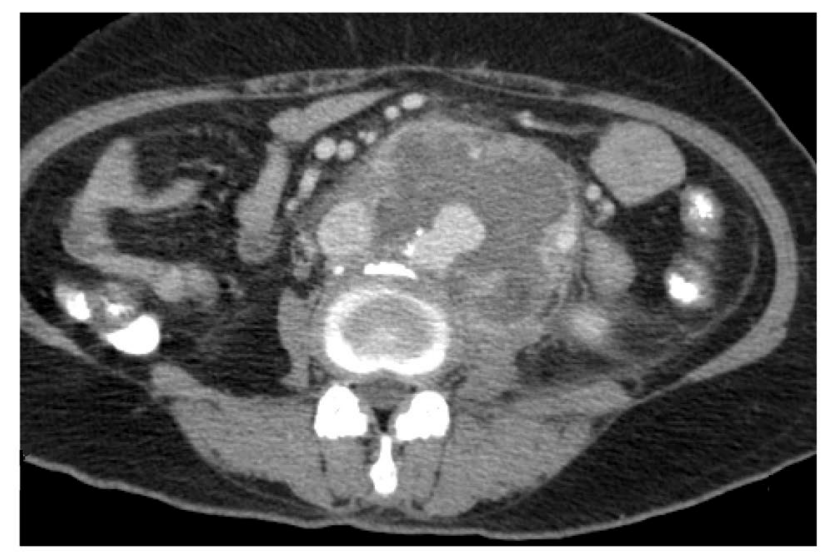

a.

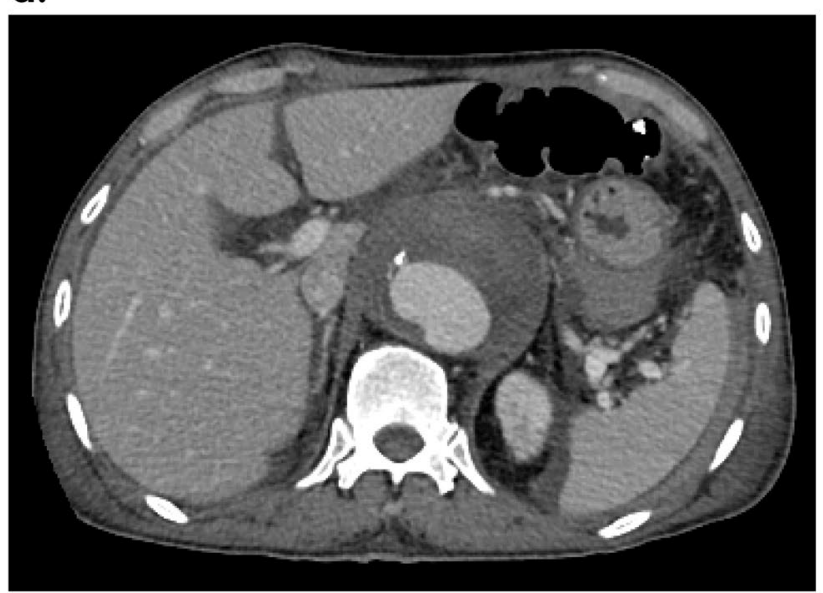

c.

Fig. 5 Periaortic CT findings of INAA. a Axial CECT image of a 58-year-old female patient demonstrates enhancing periaortic soft-tissue mass and fat stranding surrounding the multilobulated infrarenal aneurysm. $\mathbf{b}$ Axial CECT image of a 69-year-old male patient reveals a group of periaortic lymph nodes and fat stranding adjacent to the level of an infrarenal aneurysm. Left perinephric space involvement of INAA is also evident. c Axial CECT image of a 57-year-old female patient reveals a suprarenal aneurysm with periaortic soft-tissue mass and surrounding hypodense fluid extending to the peritoneal and retroperitoneal spaces. $\mathbf{d}$ Axial CECT image of a 62-year-old male patient shows periaortic soft-tissue mass with a few ectopic gas bubbles in the infrarenal aneurysm. INAA infective native aortic aneurysm, CECT contrast-enhanced computer tomography 
Table 4 Features associated with the surrounding structures of 132 aneurysms on initial CT of 114 patients with INAA

\begin{tabular}{lll}
\hline Location of INAA & $\begin{array}{l}\text { Abdominal aortic } \\
\text { aneurysm } \\
(\boldsymbol{n}=107)\end{array}$ & $\begin{array}{l}\text { Thoracic } \\
\text { aortic } \\
\text { aneurysm } \\
(\boldsymbol{n}=\mathbf{2 5})\end{array}$ \\
\hline $\begin{array}{l}\text { CT findings } \\
\text { Psoas involvement }\end{array}$ & $19(17.8)$ & - \\
Perinephric involvement & $3(2.8)$ & - \\
Aortocaval fistula & $1(0.9)$ & - \\
Hollow viscus involvement & & - \\
Bowel & $2(1.9)$ & $4(16)$ \\
Bronchus & - & $5(20)$ \\
Esophagus & - & \\
Spondylitis & $15(11.4)$ &
\end{tabular}

Data are presented as number (\%)

INAA infective native aortic aneurysm

respectively. Spondylitis was the surrounding structural involvement regardless of the INAA location found in $11.4 \%$ (15/132) (Fig. 6a).

\section{Discussion}

This is the largest study on INAA patients to report detailed findings using CT $[1,10]$. The main findings of this study were that the majority of patients had saccular aneurysms often with a multilobulated contour and an infrarenal aortic location. Also, as opposed to prior conception, many of these aneurysms contained atherosclerotic plaques.

Although calcification at the aneurysmal wall is commonly found in degenerative aortic aneurysms, it has been rarely reported in INAA, which seems to be an errant misconception $[1,10]$. Several pathological studies of INAA have shown acute transmural inflammation superimposed on the atherosclerotic aorta as the typical pathogenesis of INAA $[11,12]$, thereby supporting calcified plaque as its common CT feature. Therefore, the presence of calcified plaque could not distinguish INAA apart from a degenerative aortic aneurysm [1]. Moreover, the infrarenal aorta has been the most common site of both INAA and degenerative aortic aneurysm, inferring that INAA is a disease commonly affecting elderly patients with aged aortas and comorbidities causing atherosclerosis $[2,6,13]$.

The inflammation process involving the compromised aortic wall would also cause an aggressive appearance of the aneurysm including the saccular shape, multilobulated contour, large initial aneurysm sac, and rapid progression in size as the primary CT features of INAA $[1,10,14,15]$. Furthermore, evidence of aneurysm instability (i.e., impending rupture, contained rupture, or free rupture) from the CT findings was also found in more than half of the patients with INAA.

The typical periaortic characteristics of INAA in this study were soft-tissue mass, enhancement, fluid, and fat stranding, which were likely secondary to the infection and subsequent inflammation $[1,8,10,16-18]$.

Although some CT findings, including periaortic fluid and ectopic gas, were previously described as uncommon $[1,8,10,19-21]$, periaortic lymphadenopathy, which supported the diagnosis of INAA, was found in two-thirds of the patients in this study. However, periaortic lymphadenopathy was reported in only a few previous studies [14, 17]. Since lymphadenopathy has been one of the characteristics to indicate surrounding active infection/inflammation, its role in determining the activity of infection and the treatment response of INAA could be another opportunity for future researchers [22].

The features associated with the surrounding structures were less often noticed. However, they could be found as INAA culminated in secondary complications and thus were noteworthy for urgent management. The psoas muscle could either have been the primary site of abscess or hematoma secondary to a ruptured INAA [1, 23, 24]. These etiologies can be indistinguishable on CT, which leaves percutaneous aspiration as the most appropriate means to simultaneously treat and secure a specimen for culture and polymerase chain reaction analysis and to differentiate the two conditions from each other $[25,26]$.

Despite the rare incidence, ectopic gas should raise suspicion of a gastrointestinal structure or bronchus involvement apart from being evidence of infection [18, 23, 27]. However, contrast extravasation from INAA into a hollow viscus was rarely seen on $\mathrm{CT}$ in this study. Having said that, a definite diagnosis of aortic fistula prior to exsanguination is only possible from CT findings when appropriate clinical information, such as herald bleeding, is present [28, 29]. Also, thoracic INAA is relatively infrequent compared to abdominal INAA [30, $31]$. On the contrary, there is a greater proportion of hollow viscus complications from esophagus and bronchus involvement in thoracic INAA than bowel involvement in abdominal INAA in this study. Spondylitis and perinephric involvement were also uncommon concomitants, as reported in previous studies [1, 10, 23, 32, 33]. Only a few previous cases of aortocaval fistula in the acute setting of INAA have been reported [10,23].

Our study had some limitations. Even though this is the largest study of CT characteristics of INAA, the number of cases is relatively small due to the rare prevalence. The high rates of previous antibiotic treatment before obtaining blood culture were responsible for the low rate of positive blood culture results in this study. However, 

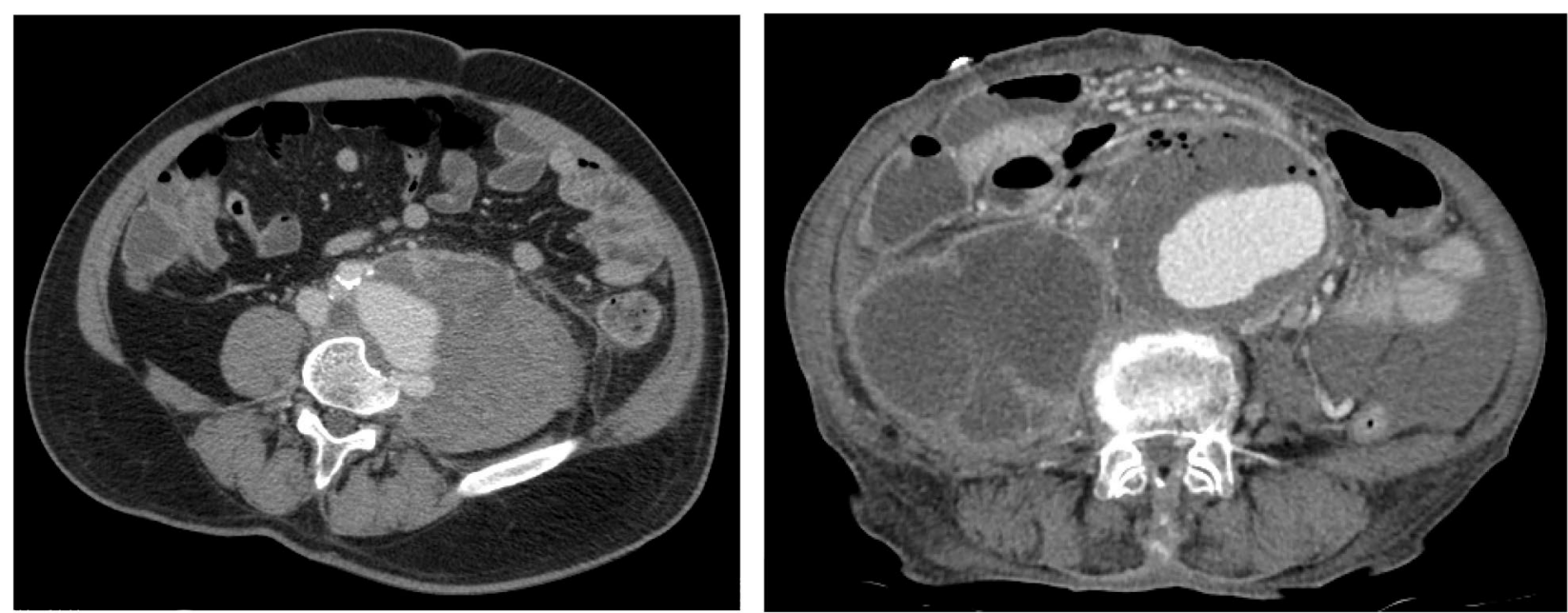

a.

b.
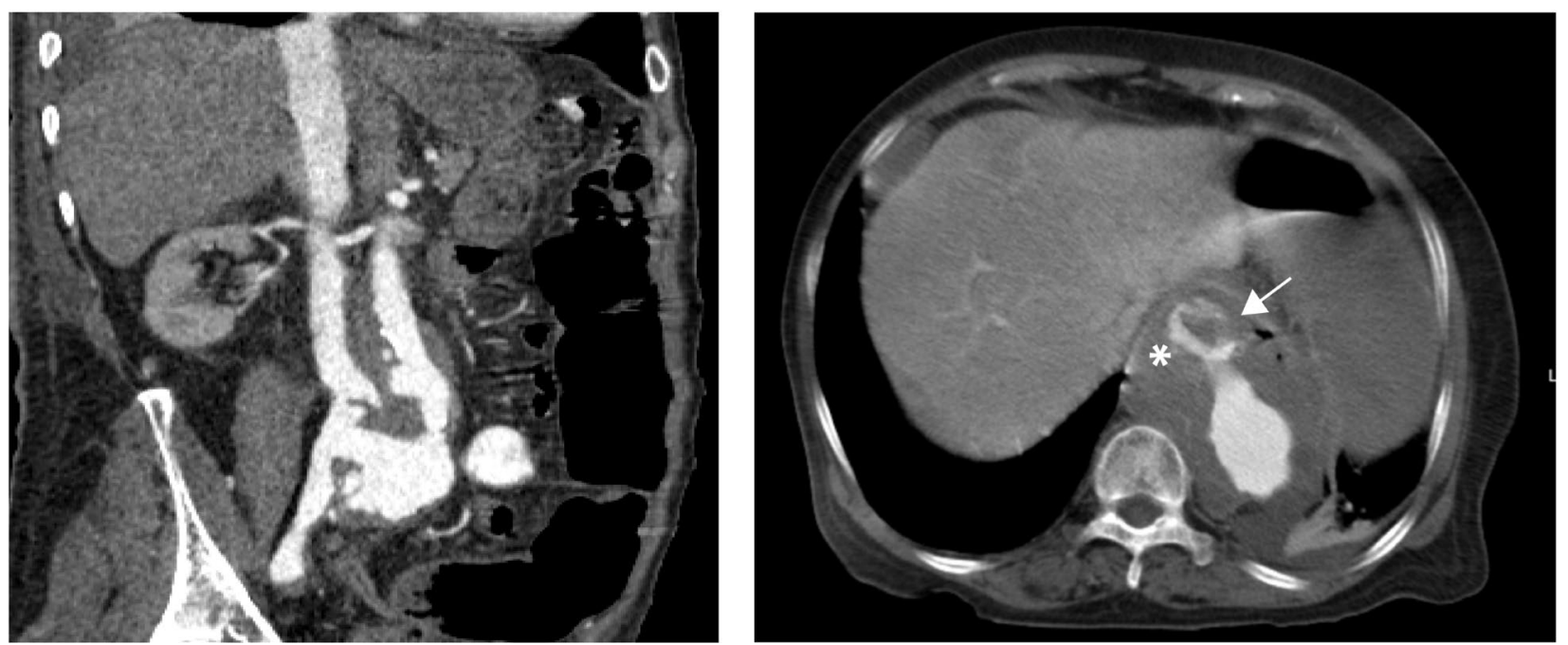

C.

d.

Fig. 6 Surrounding organ involvement of INAA. a Axial CECT of a 58-year-old male patient depicts an infrarenal aneurysm with enhancing periaortic soft-tissue mass involving the left psoas muscle. The concave eroded anterior border of the adjacent vertebral body represents spondylitis. $\mathbf{b}$ Axial CECT of an 84-year-old female patient presenting with gastrointestinal hemorrhage reveals multiple air bubbles in the infrarenal aneurysm connecting to the adjacent duodenum, suggestive of aortoenteric fistula. A rim-enhancing fluid collection involving enlarged right psoas muscle is also noted. c Coronal angiographic CT image of a 66-year-old male patient reveals early enhancement of the IVC connecting to the aorta via multilobulated aortoiliac aneurysm, suggestive of aortocaval fistula. $\mathbf{d}$ Axial CECT of an 84-year-old female patient reveals active contrast extravasation (white arrow) from INAA into the esophagus. Asterisk $\left(^{*}\right)$ indicates aortoesophageal fistula. INAA infective native aortic aneurysm, CECT contrast-enhanced computer tomography, IVC inferior vena cava

the rate of negative cultures in this study was in line with the literature [2, 34]. Maximal diameter from the outer border to the outer border of the aneurysm on axial CT image was the standard measurement method in this study. Such simplicity led to a good comparison to determine treatment response in the follow-up examinations. However, to date no standard measurement of INAA sac size has been proposed [16, 35].
In conclusion, infective native aortic aneurysms most commonly occur in the infrarenal aorta and are usually associated with calcified atherosclerotic plaques. The most common aneurysmal features are saccular shape, multilobulated contour, aneurysmal instability, and rapid expansion. The typical periaortic findings are enhancement, fat stranding, and soft-tissue mass. Involvement of the adjacent psoas muscle, gastrointestinal structures, 
and bronchi are infrequent but do occur as critical complications to INAA.

\section{Abbreviations}

CT: Computed tomography; INAA: Infective native aortic aneurysm; IVC: Inferior vena cava; MDCT: Multidetector computed tomography; SD: Standard deviation.

\section{Acknowledgements}

We gratefully thank Asst. Prof. Dr. Rassamee Sangthong from Epidemiology Unit, Faculty of Medicine, Prince of Songkla University, Thailand, for her part on the statistical analysis and manuscript editing, and Mr. Glenn Shingledecker for his assistance with the language proofreading in this manuscript.

\section{Authors' contributions}

Conception and design: WJ, RS, KS; analysis and interpretation: WJ, RS; data collection: WJ, RS; writing the article: WJ, RS, KS, KH, BK; critical revision of the article: WJ, RS, KS; final approval of the article: WJ, RS, KS; overall responsibility: RS. All authors read and approved the final manuscript.

\section{Funding}

The authors state that this work has not received any funding.

\section{Availability of data and materials}

The data that support the findings of this study are available on request from the corresponding author, (RS). The data are not publicly available due to restrictions, e.g., they contain information that could compromise the privacy of research participants.

\section{Declarations}

\section{Ethics approval and consent to participate}

The institutional review board of the Faculty of Medicine, Prince of Songkla University, approved the study (REC.64-055-7-4). Written informed consent was waived by the Institutional Review Board due to the retrospective nature of the study.

\section{Consent for publication}

All images are entirely unidentifiable, and there are no details on individuals reported within the manuscript.

\section{Competing interests}

The authors declare that they have no competing interests.

\section{Author details}

${ }^{1}$ Department of Radiology, Faculty of Medicine, Prince of Songkla University, 15 Karnjanavanich Rd., Hat Yai, Songkhla 90110, Thailand. ${ }^{2}$ Department of Surgery, Faculty of Medicine, Prince of Songkla University, 15 Karnjanavanich Rd, Hat Yai, Songkhla 90110, Thailand. ${ }^{3}$ Department of Vascular Surgery, Rigshospitalet, Copenhagen, and Faculty of Health and Medical Sciences, University of Copenhagen, Copenhagen, Denmark.

\section{Received: 5 October 2021 Accepted: 26 November 2021}

Published online: 08 January 2022

\section{References}

1. Macedo TA, Stanson AW, Oderich GS, Johnson CM, Panneton JM, Tie ML (2004) Infected aortic aneurysms: imaging findings. Radiology 231(1):250-257

2. Sörelius K, Budtz-Lilly J, Mani K, Wanhainen A (2019) Systematic review of the management of mycotic aortic aneurysms. Eur J Vasc Endovasc Surg 58(3):426-435
3. Sörelius K, Wanhainen A, Mani K (2020) Infective native aortic aneurysms: call for consensus on definition, terminology, diagnostic criteria, and reporting standards. Eur J Vasc Endovasc Surg 59(3):333-334

4. Wilson SE, Gordon HE, Van Wagenen PB (1978) Salmonella arteritis: a precursor of aortic rupture and pseudoaneurysm formation. Arch Surg 113(10):1163-1166

5. Shang EK, Nathan DP, Boonn WW et al (2013) A modern experience with saccular aortic aneurysms. J Vasc Surg 57(1):84-88

6. Isselbacher EM (2005) Thoracic and abdominal aortic aneurysms. Circulation 111(6):816-828

7. Wanhainen A, Verzini F, Van Herzeele I et al (2019) Editor's choice-European Society for Vascular Surgery (ESVS) 2019 clinical practice guidelines on the management of abdominal aorto-iliac artery aneurysms. Eur J Vasc Endovasc Surg 57(1):8-93

8. Sörelius K, Mani K, Björck M et al (2014) Endovascular treatment of mycotic aortic aneurysms: a European multicenter study. Circulation 130(24):2136-42

9. Restrepo CS, Ocazionez D, Suri R, Vargas D (2011) Aortitis: imaging spectrum of the infectious and inflammatory conditions of the aorta. Radiographics 31(2):435-451

10. Lee W-K, Mossop PJ, Little AF et al (2008) Infected (Mycotic) aneurysms: spectrum of imaging appearances and management. Radiographics 28(7):1853-1868

11. Miller DV, Oderich GS, Aubry M-C, Panneton JM, Edwards WD (2004) Surgical pathology of infected aneurysms of the descending thoracic and abdominal aorta: clinicopathologic correlations in 29 cases (1976 to 1999). Hum Pathol 35(9):1112-1120

12. Hsu R-B, Lin F-Y (2007) Surgical pathology of infected aortic aneurysm and its clinical correlation. Ann Vasc Surg 21(6):742-748

13. Norman PE, Powell JT (2010) Site specificity of aneurysmal disease. Circulation 121(4):560-568

14. Rozenblit A, Bennett J, Suggs W (1996) Evolution of the infected abdominal aortic aneurysm: CT observation of early aortitis. Abdom Imaging 21(6):512-514

15. Gonda RL, Gutierrez OH, Azodo MV (1988) Mycotic aneurysms of the aorta: radiologic features. Radiology 168(2):343-346

16. Sörelius K, di Summa PG (2018) On the diagnosis of mycotic aortic aneurysms. Clin Med Insights Cardiol 12:1179546818759678

17. Sueyoshi E, Sakamoto I, Kawahara Y, Matsuoka Y, Hayashi K (1998) Infected abdominal aortic aneurysm: early CT findings. Abdom Imaging 23(6):645-648

18. Azizi L, Henon A, Belkacem A, Monnier-Cholley L, Tubiana J-M, Arrivé $L$ (2004) Infected aortic aneurysms: CT features. Abdom Imaging 29(6):716-720

19. Sörelius K, Wanhainen A, Furebring M et al (2016) Nationwide study of the treatment of mycotic abdominal aortic aneurysms comparing open and endovascular repair. Circulation 134(23):1822-32.

20. Sörelius K, Mani K, Björck M, Nyman R, Wanhainen A (2009) Endovascular repair of mycotic aortic aneurysms. J Vasc Surg 50(2):269-274

21. Sörelius K, Wanhainen A, Wahlgren CM et al (2019) Nationwide study on treatment of mycotic thoracic aortic aneurysms. Eur J Vasc Endovasc Surg 57(2):239-246

22. Einstein DM, Singer AA, Chilcote WA, Desai RK (1991) Abdominal lymphadenopathy: spectrum of CT findings. Radiographics 11(3):457-472

23. Sessa C, Farah I, Voirin L, Magne J-L, Brion J-P, Guidicelli H (1997) Infected aneurysms of the infrarenal abdominal aorta: diagnostic criteria and therapeutic strategy. Ann Vasc Surg 11(5):453-463

24. Hsu R-B, Lin F-Y (2007) Psoas abscess in patients with an infected aortic aneurysm. J Vasc Surg 46(2):230-235

25. Torres G, Cernigliaro J, Abbitt P et al (1995) Iliopsoas compartment: normal anatomy and pathologic processes. Radiographics 15:1285-1297

26. Muttarak M, Peh WCG (2000) CT of unusual iliopsoas compartment lesions. Radiographics 20(suppl_1):S53-66

27. Gomes MN, Choyke PL, Wallace RB (1992) Infected aortic aneurysms. A changing entity Ann Surg 215(5):435-442

28. Hagspiel KD, Turba UC, Bozlar U et al (2007) Diagnosis of aortoenteric fistulas with CT angiography. J Vasc Intervent Radiol 18(4):497-504

29. Vu QDM, Menias CO, Bhalla S, Peterson C, Wang LL, Balfe DM (2009) Aortoenteric fistulas: CT features and potential mimics. Radiographics 29(1):197-209 
30. Jonker FHW, Heijmen R, Trimarchi S, Verhagen HJM, Moll FL, Muhs BE (2009) Acute management of aortobronchial and aortoesophageal fistulas using thoracic endovascular aortic repair. JVasc Surg 50(5):999-1004

31. Pickhardt PJ, Bhalla S, Balfe DM (2002) Acquired gastrointestinal fistulas: classification, etiologies, and imaging evaluation. Radiology 224(1):9-23

32. Vogelzang RL, Sohaey R (1988) Infected aortic aneurysms: CT appearance. J Comput Assist Tomogr 12(1):109-112

33. McHenry MC, Rehm SJ, Krajewski LP, Duchesneau PM, Levin HS, Steinmuller DR (1991) Vertebral osteomyelitis and aortic lesions: case report and review. Rev Infect Dis 13(6):1184-1194

34. Dang Q, van Eps RGS, Wever JJ et al (2020) Nationwide study of the treatment of mycotic abdominal aortic aneurysms comparing open and endovascular repair in The Netherlands. J Vasc Surg 72(2):531-540

35. Wang TKM, Griffin B, Cremer P et al (2020) Diagnostic utility of CT and MRI for mycotic aneurysms: a meta-analysis. AJR Am J Roentgenol 215(5):1257-1266

\section{Publisher's Note}

Springer Nature remains neutral with regard to jurisdictional claims in published maps and institutional affiliations.

\section{Submit your manuscript to a SpringerOpen ${ }^{\odot}$ journal and benefit from:}

- Convenient online submission

- Rigorous peer review

- Open access: articles freely available online

- High visibility within the field

- Retaining the copyright to your article

Submit your next manuscript at $\boldsymbol{\nabla}$ springeropen.com 